\section{Silicon waveguide based nonlinear directional coupler as a soliton switch}

Amarendra K. Sarma

Indian Institute of Technology Guwahati, Department of Physics, Guwahati-781039, India

E-mail: aksarma@iitg.ernet.in

\begin{abstract}
We propose, for the first time to the best of our knowledge, a design for a silicon waveguide-based nonlinear directional coupler in order to achieve soliton selfswitching. Numerical analysis shows that the proposed design may be quite useful as a soliton switch. @ 2008 Society of Photo-Optical Instrumentation Engineers.

[DOI: 10.1117/1.3041656]
\end{abstract}

Subject terms: silicon photonics; optical soliton; soliton switching.

Paper 080599LR received Jul. 31, 2008; revised manuscript received Oct. 28, 2008; accepted for publication Oct. 29, 2008; published online Dec. 12, 2008.

Silicon-on-insulator (SOI) waveguides have attracted considerable attention recently because they can be used for making inexpensive, monolithically integrated optical devices. ${ }^{1-4}$ The most notable features of silicon waveguides are that silicon exhibits significant third-order nonlinearity and provides a tight mode confinement. These features make it possible to realize a variety of optical functions at relatively low power level. Recently, the formation of optical solitons inside a short silicon waveguide is reported. ${ }^{5}$ Solitons have been observed inside silica fibers for a long time and found a multitude of applications ranging from optical switching to supercontinuum generation. ${ }^{6}$ In this letter, we propose, for the first time to our knowledge, a novel design of a silicon waveguide-based nonlinear directional coupler (NLDC), which can be used as a soliton self-switching device. After the pioneering work of Jensen ${ }^{7}$ and Trillo et al. ${ }^{8}$ silica based nonlinear directional couplers have been studied quite extensively ${ }^{9-21}$ in the context of soliton switching. Jensen ${ }^{7}$ showed that one can switch a continuous signal from one core to the other by varying the input power of the signal. The idea when applied to pulse switching led to pulse distortion and breakup, resulting in inefficient switching. Because the nonlinear phase modulation is proportional to the instantaneous intensity, different portions of the pulse envelope switch differently (i.e., not simultaneously), leading to pulse distortion and pulse breakup. The pulse breakup during switching is undesirable because it results in inefficient switching and causes cross talk of the signals. Trillo et al. ${ }^{8}$ showed that pulse breakup could be avoided if one used a soliton pulse as a signal. The physics behind it can be understood from the fact that the nonlinear phase modulation is constant across the entire soliton pulse, owing to which the pulse switches as a whole (i.e., as a single unit and no pulse breakup takes place). However, because of silica's weak nonlinearity, extremely high power is needed for soliton switching in silica-based NLDC. This difficulty in experimental realization has left

0091-3286/2008/\$25.00 @ 2008 SPIE the silica-based NLDC as only a device of theoretical interest. On the other hand, silicon waveguide-based NLDC should not have this drawback because the nonlinearity parameter in the SOI waveguide can be larger by a factor of 10,000 or more. ${ }^{5}$ We expect that our proposed design for a silicon waveguide-based NLDC would draw the attention of the experimentalists working in this field. It should be noted that soliton self-switching can also be achieved by applying a different scheme in which the switching is controlled by copropagating a controlling pulse along with the signal pulse, for a detailed discussion the readers are referred to Refs. 20 and 21.

It is well known that the formation of fundamental soliton requires $L_{\mathrm{D}}=L_{\mathrm{N}}$ where $L_{\mathrm{D}}=T_{0}^{2} /\left|\beta_{2}\right|$, the dispersion length, and $L_{\mathrm{N}}=1 / \gamma_{0} P_{0}$, the nonlinear length. It has been shown that because of high nonlinearity and tight mode confinement in SOI waveguides both $\gamma_{0}$ and $\beta_{2}$ are sufficiently large to support optical solitons. Figure 1 shows our proposed NLDC. Here, two identical silicon waveguides are placed close to each other. The individual waveguides have the same dimensions as that of Zhang et al.'s (i.e., of $\left.860 \times 400 \mathrm{~nm}^{2}\right) .^{5}$ The core-to-core separation distance is $2.4 \mu \mathrm{m}$. The coupling coefficient can be calculated by following the prescription in Ref. 22. For our proposed NLDC, it is calculated to be $2.38 \mathrm{~m}^{-1}$. The core-to-core distance is extremely crucial in deciding the coupling coefficient of the coupler. It will be seen shortly that the coupling coefficient plays the most significant role in soliton switching in an NLDC. The switching characteristics of the coupler are described by the generalized coupled nonlinear Schrodinger equation in the anomalous dispersion regime (i.e., $\beta_{2}<0$ ), which can be derived in the framework of the coupled mode formalism, using the standard slowly varying envelope approximation, ${ }^{6}$

$$
\begin{aligned}
& \frac{\partial A_{1}}{\partial z}+\frac{\alpha}{2} A_{1}+\frac{i}{2} \beta_{2} \frac{\partial^{2} A_{1}}{\partial T^{2}}-i \gamma\left|A_{1}\right|^{2} A_{1}+C_{0} A_{2}=0, \\
& \frac{\partial A_{2}}{\partial z}+\frac{\alpha}{2} A_{2}+\frac{i}{2} \beta_{2} \frac{\partial^{2} A_{2}}{\partial T^{2}}-i \gamma\left|A_{2}\right|^{2} A_{2}+C_{0} A_{1}=0 .
\end{aligned}
$$

Here, $A_{1}$ and $A_{2}$ are the slowly varying pulse envelopes in core 1 and core 2 , respectively. $\gamma=\gamma_{0}(1+i r)$ is the nonlinear parameter where the dimensionless parameter $r$ includes two-photon absorption. $\alpha$ takes into account the linear scattering loss. $\beta_{2}$ is the second-order dispersion

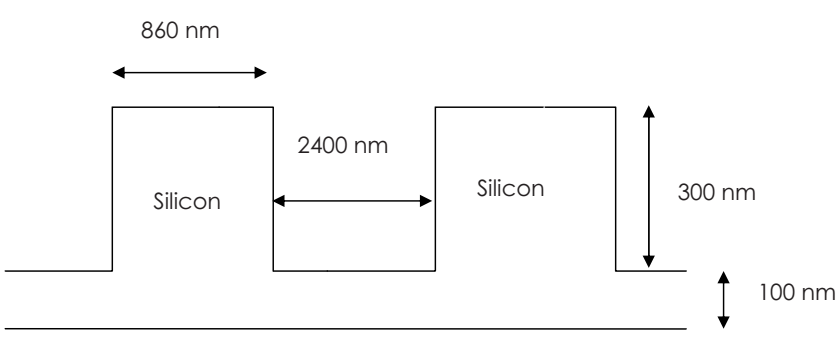

Silicon oxide

Fig. 1 Design of silicon waveguide based nonlinear directional coupler. 


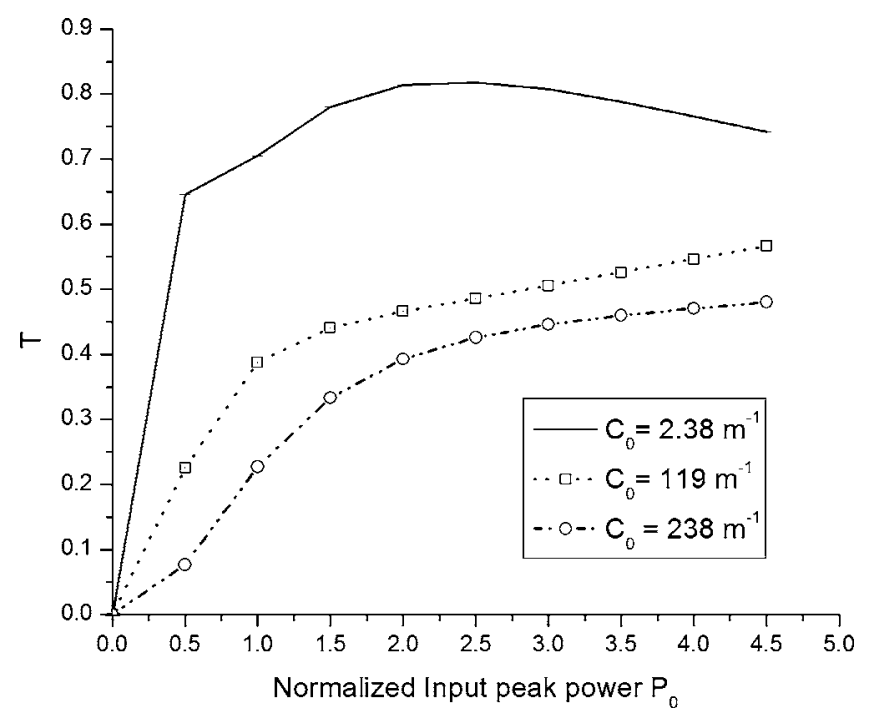

Fig. 2 Plot of the transmission coefficient as a function of the normalized input peak power.

parameter, and $C_{0}$ is the coupling coefficient. A parameter of utmost importance in switching literature is the so-called coupling length $L_{C}=\pi / 2 C_{0}$ for a $\pi / 2$ coupler, which is defined as the length at which the power completely transfers from the input fiber to the other fiber. Because the set of coupled Eqs. (1) and (2) is not analytically solvable, we solve them numerically by the fast Fourier transform method for the linear dispersive part and by the fourthorder Runge-Kutta method, for the nonlinear part, with autocontrol of the step size for a given accuracy of the results. We calculate the transmission coefficient $T$, representing the fractional output energy in core 1 , according to the formula

$T=\frac{\int_{-\infty}^{\infty}\left|u_{1}(\xi, \tau)\right|^{2} d \tau}{\int_{-\infty}^{\infty}\left[\left|u_{1}(\xi, \tau)\right|^{2}+\left|u_{2}(\xi, \tau)\right|^{2}\right] d \tau}$.

Here, $u_{1}$ and $u_{2}$ are the normalized pulse envelopes in core 1 and core 2 , respectively. $\xi=z / L_{\mathrm{D}}$ and $\tau=T / T_{0}$ are normalized distance and time, respectively. In this work, we have calculated the transmission coefficient $T$ at the end of one coupling length of the coupler. To analyze the switching process we consider the following initial conditions:

$u_{1}(0, \tau)=\sqrt{P_{0}} \sec h\left(\sqrt{P_{0}} \tau\right)$,

$u_{2}(0, \tau)=0$.

We have used the following parameters for our analysis: ${ }^{5}$ $\beta_{2}=-2.15 \mathrm{ps}^{2} / \mathrm{m}, n_{2}=6 \times 10^{-5} \mathrm{~cm}^{2} / \mathrm{GW}, \alpha=2.30 \mathrm{~m}^{-1}, r$ $=0.1, \lambda_{0}=1484 \mathrm{~nm}$, and $T_{0}=30 \mathrm{fs}$.

Figure 2 depicts the transmission characteristics of the coupler as a function of the normalized peak power $P_{0}$ for three different values of the coupling coefficients: $C_{0}$ $=2.38,119$, and $238 \mathrm{~m}^{-1}$. These coupling coefficients can be achieved quite easily just by varying the core-to-core distance of the coupler. It can be seen from Fig. 2 that $C_{0}$

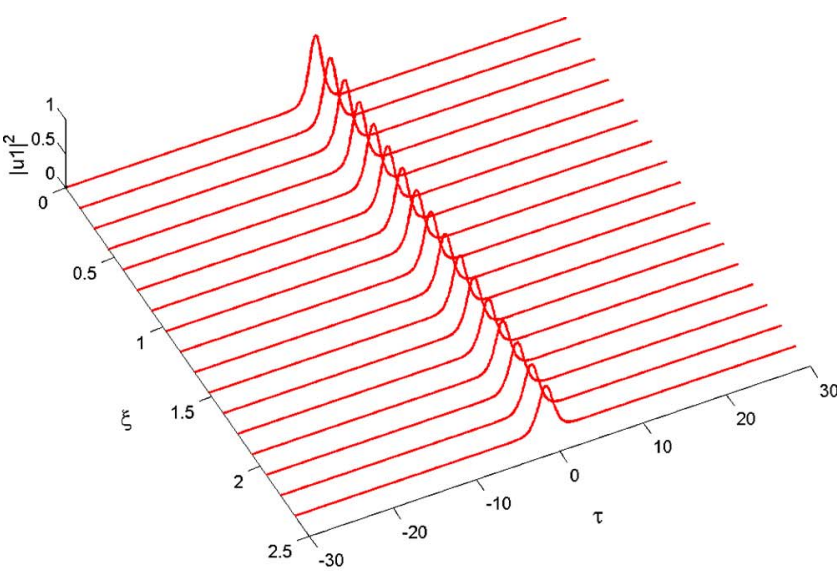

(a)

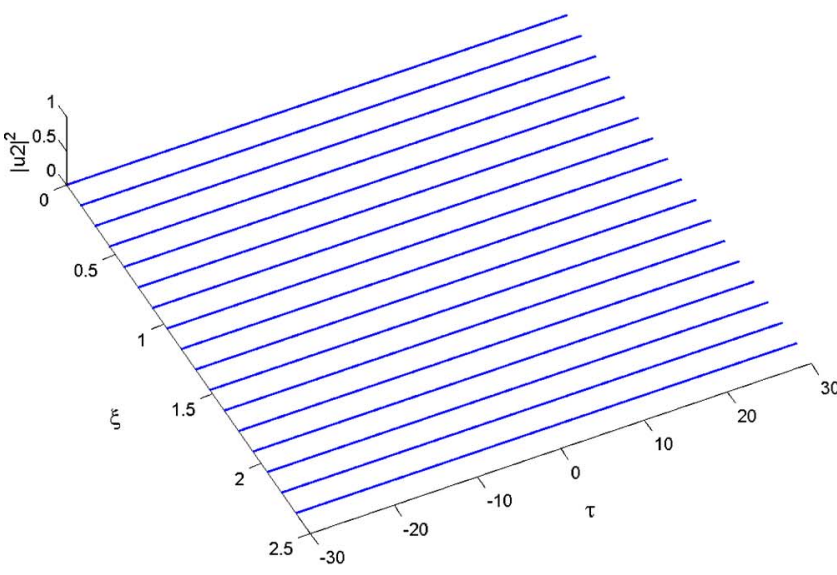

(b)

Fig. 3 (a) Spatiotemporal evolution of the soliton pulse inside the coupler in core 1 and (b) spatiotemporal evolution of the radiation inside the coupler in core 2 .

$=2.38 \mathrm{~m}^{-1}$ gives the best transmission. At $P_{0} \approx 2$, we get nearly $80 \%$ transmissions. As the coupling coefficient or equivalently the core-to-core separation is increased soliton switching may not be realized in our proposed device. To get an idea about the behavior and stability of the soliton pulse inside the coupler in Fig. 3, we depict the spatiotemporal evolution of the soliton pulse inside the coupler. It can clearly be seen that the soliton is preserved during evolution inside core 1 , while at the output of core 2 we may get a negligibly small radiation, confirming that the soliton is getting completely switched to the bar state of the coupler. It is worthwhile to mention that the peak power of the soliton required for switching in our proposed device is calculated to be $12 \mathrm{~W}$, which is clearly an improvement over that of the corresponding silica based NLDC where the switching power is generally in the range of kilowatts. ${ }^{14,18}$

\section{References}

1. R. Soref, "The past, present, and future of silicon photonics," IEEE $J$ Sel. Top. Quantum Electron. 12, 1678-1687 (2006).

2. V. R. Almeida, C. A. Barrios, R. R. Panepucci, and M. Lipson, "Alloptical control of light on a silicon chip," Nature (London) $\mathbf{4 3 1}$ 1081-1084 (2004) 
3. H. Rong, R. Jones, A. Liu, O. Cohen, D. Hak, A. Fang, and M. Paniccia, "A continuous-wave Raman silicon laser," Nature (London) 433, 725-728 (2005)

4. M. A. Foster, A. C. Turner, J. E. Sharping, B. S. Schmidt, M. Lipson, and A. L. Gaeta, "Broad-band optical parametric gain on a silicon photonic chip," Nature (London) 441, 960-963 (2006).

5. J. Zhang, Q. Lin, G. Piredda, R. W. Boyd, G. P. Agrawal, and P. M Fauchet, "Optical solitons in a silicon waveguide," Opt. Express 15 $7682-7688$ (2007)

6. G. P. Agrawal, Nonlinear Fiber Optics, 4th ed., Academic Press, Boston (2007)

7. S. M. Jensen, "Nonlinear coherent coupler," IEEE J. Quantum Electron. 18, 1580-1583 (1982).

8. S. Trillo, S. Wabnitz, E. M. Wright, and G. I. Stegeman, "Soliton switching in fiber nonlinear directional couplers," Opt. Lett. 13, 672 674 (1988)

9. P. M. Ramos and C. R. Paiva "All-optical pulse switching in twincore fiber couplers with intermodal dispersion," IEEE J. Quantum Electron. 35, 983-989 (1999).

10. A. Kumar and A. K. Sarma, "Soliton switching in a Kerr coupler with coupling constant dispersion: A variational analysis," Opt. Commun. 234, 427-432 (2004)

11. G. I. Stegeman and E. M. Wright, "All-optical waveguide switching," Opt. Quantum Electron. 22, 95-98 (1990).

12. N. Finlayson and G. I. Stegeman, "Spatial switching, instabilities, and chaos in a three-waveguide nonlinear directional coupler," Appl. Phys. Lett. 56, 2276-2278 (1990).
13. Y. Wang and W. Wang, "Study of ultrafast pulse coupling dynamics considering retarded nonlinear response and self-steepening effects," J. Lightwave Technol. 24, 1041-1047 (2006).

14. A. Kumar and A. K. Sarma, "Femtosecond-soliton switching in a three-core coupler," Jpn. J. Appl. Phys., Part 1 44, 8498-8500 (2005).

15. I. M. Skinner, G. D. Peng, B. A. Malomed, and P. L. Chu, "Soliton switching in a variable nonlinear coupler," Opt. Commun. 113, 493497 (1995).

16. B. A. Umarov, F. Kh. Abdullaev, and M. K. B. Wahiddin, "Soliton interaction and switching in a coupler with third order dispersion and Raman effect," Opt. Commun. 162, 340-346 (1999).

17. B. A. Malomed, I. M. Skinner, and S. Tasgal, "Solitons in a nonlinear optical coupler in the presence of the Raman effect," Opt. Commun. 139, 247-251 (1997)

18. A. K. Sarma and A. Kumar, "Phase induced soliton switching in fiber nonlinear directional couplers," Opt. Eng. 46(11), 115005 (2007).

19. A. K. Sarma, "Soliton self-switching in an asymmetric nonlinear directional coupler," J. Optoelectron. Adv. Mater. 11, 558-562 (2007).

20. W. S. Main, M. S. Demokan, and H. Tam, "All-optical routing switch using a fiber nonlinear directional coupler and controlling solitons," J. Lightwave Technol. 14, 2793-2798 (1996).

21. N. Trivunac-Vukovic and P. Vukovic, "Ultrafast all-optical switching using controlling solitons and nonlinear directional coupler," in TELSIKS'99, pp. 345-348 (1999).

22. A. W. Snyder and J. D. Love, Optical Waveguide Theory, Chaps 12-15, Chapman and Hall, London (1983). 